\title{
Temperature Dependence of Internal Torsional Frequencies in 3,4-Dichloro Nitrobenzene and 4-Chloro 2 Nitrophenol Using NQR Data
}

\author{
K. Rukmani* and J. Jesu Jamila Rani \\ Department of Physics, Bangalore University, Bangalore 560056, India
}

(Received July 14, 2009; revised version September 14, 2009; in final form October 1, 2009)

\begin{abstract}
The temperature variation of the nuclear quadrupole resonance frequency in the two compounds 3,4-dichloro nitrobenzene and 4-chloro 2 nitrophenol were analyzed in the light of the Kushida and Brown models. The internal torsional frequencies at each temperature was calculated using Bayer's model with Tatsuzaki correction under a two-mode approximation to obtain the temperature variation of the torsional frequencies in both compounds. The torsional frequencies are seen to lie between $25 \mathrm{~cm}^{-1}$ and $85 \mathrm{~cm}^{-1}$ in the first compound and between $30 \mathrm{~cm}^{-1}$ and $42 \mathrm{~cm}^{-1}$ in the case of the second compound. The temperature dependence of the torsional frequencies is linear above $150 \mathrm{~K}$ in the first compound and is linear throughout the range $77 \mathrm{~K}$ to $350 \mathrm{~K}$ in the second. The smaller temperature dependence in the second compound may be the effect of hydrogen bonding in this compound.
\end{abstract}

PACS numbers: $76.60 \mathrm{Gv}$

\section{Introduction}

The observation of pure nuclear quadrupole resonance (NQR) in chlorine substituted benzene derivatives has been of interest for many years [1,2] and the temperature dependence of the ${ }^{35} \mathrm{Cl} \mathrm{NQR}$ frequency reveals details of the internal motions and structural phase transitions if any. In general, the ${ }^{35} \mathrm{Cl} \mathrm{NQR}$ frequency increases with decreasing temperature and this is understood on the basis of Bayer's theory [3] with its many modifications [4-6]. Since the internal torsional motions are assumed to give rise to the temperature dependence of the NQR frequency, it is possible to obtain features of the internal motions from the NQR temperature dependence [7-9]. It is known that the internal torsional frequencies whose effect is seen on the NQR temperature dependence are the low frequency modes mainly below $150 \mathrm{~cm}^{-1}$. These modes can be calculated theoretically under some approximations or obtained experimentally as direct measurements in the Raman laser spectroscopy. However, obtaining the low frequency modes, say below $200 \mathrm{~cm}^{-1}$, by the Raman laser method is difficult as the central line swamps this region. It is possible to obtain these low frequency modes from the experimental NQR data and in the case of the dichloro anilines [9] it was seen that the modes obtained by this method agreed closely with those observed by the Raman spectroscopy.

\footnotetext{
* corresponding author; rukmani9909@yahoo.co.in
}

The chloro nitro benzenes have generated interest in recent times because of their use in the pharmaceutical industry. They form additives with other organic molecules such as aniline, in varying proportions, and $\mathrm{X}$-ray crystallographic studies reveal interesting ribbon-like structures in these $[10,11]$. The basic chloro nitro benzenes are small molecules and have been studied extensively by NQR in the 1970's. New applications of these and their additives in biology, provided a basis for looking at the data on these compounds again and analyzing some dynamics in them. A computer based numerical method was used and the internal torsional frequencies and their temperature dependence in 3,4-dichloro nitrobenzene and 4-chloro 2 nitrophenol, both containing nitro groups, were calculated from their NQR data. The validity of the existing theories forms the basis of this method, and the slightly different results in the two compounds give some indication of the modes which affect the NQR frequency in each case.

\section{Theory}

${ }^{35} \mathrm{Cl}$ has $I=3 / 2$ and the pure NQR in compounds containing it arises from transitions between the degenerate $\pm 1 / 2$ levels to the degenerate $\pm 3 / 2$ levels of the quadrupolar nucleus when placed in the electric field gradient arising from electron and ion charge distributions in the bonds of the molecule [12]. In benzene ring derivatives, the $\mathrm{C}-\mathrm{Cl}$ bond may be taken as the principal direction of the electric field gradient (EFG) tensor. The internal torsional frequencies in a molecule may be represented as occupation of torsional levels and the popu- 
lation in these levels vary with temperature. This population dependence with temperature may be expressed equivalently as a change in the amplitude of the motions. Amongst these, it is the bond bending motions which contribute to the change in NQR frequency with temperature. Bayer's theory [3] assumes that the bond bending motions of the molecule occur at frequencies much greater than the NQR frequency and only their average effect is reflected in the NQR frequency. According to this theory, the temperature dependence of the NQR frequency is given by

$$
\begin{aligned}
& \nu(T)=\nu_{0}\{1 \\
& \left.-\frac{3 h}{8 \pi^{2}} \sum_{i} \frac{1}{A_{i} f_{i}}\left[\frac{1}{2}+\frac{1}{\exp \left(h f_{i} / k T\right)-1}\right]\right\},
\end{aligned}
$$

where $\nu_{0}$ is the NQR frequency at $0 \mathrm{~K}, h$ is the Planck constant, $A_{i}$ are the moments of inertia, $f_{i}$ are the torsional frequencies about EFG axis, $k$ is the Boltzmann constant and $T$ is the temperature. In this equation, $i$ takes the values $x$ and $y$ corresponding to motion about the $x$ and $y$ axes of the EFG tensor as these bend the (z-axis) $\mathrm{C}-\mathrm{Cl}$ bond, giving rise to the variation in $\nu(T)$. Under the high temperature approximation $h f \ll k T$, this equation was shown by Kushida et al. [4] to have the form

$$
\nu(T)=A_{-1} / T+A_{0}+A_{1} T .
$$

Bayer's model indicates a decrease in NQR frequency with increasing temperature. This is the trend observed experimentally, but the temperature variation observed could not be explained completely by this alone. In molecular crystals, Brown [5] assumed a linear temperature dependence of the torsional frequencies, which is a small frequency change associated with temperature related volume changes in the crystal. The Raman spectra which probe these frequencies directly also indicate a small variation in frequency with temperature and representative examples are the following two papers [13, 14]. Brown assumed this small change in torsional frequency with temperature to be linear and obtained the equation

$$
\nu(T)=A_{0}+A_{1}\left(T-T_{0}\right)+A_{2}\left(T-T_{0}\right)^{2},
$$

where $T_{0}$ is an arbitrary temperature in the high temperature region. The temperature variation of the NQR frequency could also be attempted to fit a general fourth order polynomial of the form given below

$$
\nu(T)=A_{0}+A_{1} T+A_{2} T^{2}+A_{3} T^{3}+A_{4} T^{4} .
$$

The equation that fits the NQR data may indicate whether the internal torsional frequencies vary with temperature or not.

\section{Torsional mode calculations}

The temperature variation of the NQR frequency as given by Bayer's theory and Eq. (1) assumes the internal torsional vibrations to occur about the principal directions of the EFG tensor. Tatsuzaki [6] pointed out that the internal torsional motions are likely to occur about the principal axes of the moment of inertia of the molecule and modified Bayer's equation to give

$$
\begin{aligned}
& \nu(T)=\nu_{0}\{1 \\
& \left.-\frac{3 h}{8 \pi^{2} c} \sum_{i} \frac{\sin ^{2} \alpha_{i}}{A_{i} f_{i}}\left[\frac{1}{2}+\frac{1}{\exp \left(h f_{i} c / k T\right)-1}\right]\right\} .
\end{aligned}
$$

The only difference in form is a factor of $\sin ^{2} \alpha_{i}$, where $\alpha_{i}$ is the angle between the $z$-direction of the EFG and the axis of the respective moment of inertia tensor. The other constants are as in Eq. (1) with $A_{i}$ being the three moments of inertia and $f_{i}$ the corresponding torsional frequencies for motion about the moment of inertia axes. In the above Tatsuzaki equation, $i$ takes three values corresponding to the three moment of inertia directions and there are three terms in the summation with three torsional modes being considered.

The two-mode approximation $[9,15,16]$ consists in retaining the two leading terms in the summation and neglecting the third. The weight factor for each term is $\sin ^{2} \alpha_{i} / A_{i}$ with $i$ taking three values. The term corresponding to the least of these is neglected and the subscripts of the highest term is called 1 and those of the next called 2 .

Numerical calculation of $f_{1}$ and $f_{2}$ from Eq. (5) is done in the following manner. The angle $\alpha_{i}$ and the corresponding moment of inertia $A_{i}$ are known from molecular parameters. $f_{1}$ is fixed at $1 \mathrm{~cm}^{-1}$ say and $f_{2}$ varied from $1 \mathrm{~cm}^{-1}$ to $150 \mathrm{~cm}^{-1}$ in Eq. (5) to obtain values of $\nu(T)$. Next, $f_{1}$ is fixed at the next value of $2 \mathrm{~cm}^{-1}$ and $f_{2}$ varied from $2 \mathrm{~cm}^{-1}$ to $150 \mathrm{~cm}^{-1}$. Thus $f_{1}$ is varied through the entire range $1 \mathrm{~cm}^{-1}$ to $150 \mathrm{~cm}^{-1}$ and $f_{2}$ varies from $f_{1}$ to $150 \mathrm{~cm}^{-1}$ for each value. In this set of numerically generated values of $\nu(T)$ only those which match the experiment within an error of $1 \mathrm{kHz}$ are retained. The correct pair of $\left(f_{1}, f_{2}\right)$ is chosen from this set by using the following assumptions. (i) In the absence of phase transitions, both the modes $f_{1}$ and $f_{2}$ are affected similarly by temperature. (ii) The energy in each mode is equal, giving $A_{1} f_{1}^{2}=A_{2} f_{2}^{2}$ which places the constraint that the ratio of $f_{1} / f_{2}$ should be close to $\sqrt{A_{2} / A_{1}}$. Thus at a given temperature $T$, the experimental NQR frequency $\nu(T)$ is compared with the one calculated numerically by the computer by assuming combinations of $f_{1}$ and $f_{2}$ in Eq. (5). This is done for every temperature, and the pair of values $\left(f_{1}, f_{2}\right)$ that gives a calculated NQR frequency matching experimental $\nu(T)$ at each temperature is obtained, thus giving the temperature variation of the torsional frequencies.

\subsection{3,4-dichloro nitrobenzene}

Ramakrishna [17] has observed two NQR frequencies in this compound throughout the temperature range $77 \mathrm{~K}$ to $301 \mathrm{~K}$ and these can be assigned to the two chlorines in the molecule by using the additive model of the substituent effect [1]. The lower frequency line, Line 1 may be assigned to the 4-chlorine and the higher frequency line, Line 2 may be assigned to the 3 -chlorine. The results of iterative least squares fitting to Eqs. (2), (3) and (4) 
is given in Table I and Table II for Line 1 and Line 2, respectively. The parameter $\langle g\rangle$ is the average temperature coefficient of the torsional frequency obtained from the best fit corresponding to a given model and $\Delta$ is the root mean square deviation of the theoretical fit. The ac- curacy of the NQR frequency in the experiment is of the order of $1 \mathrm{kHz}$, so the calculated parameters are quoted to one order higher accuracy so as to minimize rounding off errors.

Least squares fit parameters and average temperature coefficient $\langle g\rangle$ for Line 1

TABLE I of 3,4-dichloro nitrobenzene.

\begin{tabular}{l|c|c|c}
\hline \hline \multicolumn{1}{c|}{ Parameters } & $\begin{array}{c}\text { 4th order } \\
\text { polynomial }\end{array}$ & Brown $\left(T_{0}=200 \mathrm{~K}\right)$ & Kushida \\
\hline$A_{-1}[\mathrm{MHz} \mathrm{K}]$ & - & - & $0.334 \times 10^{2}$ \\
$A_{0}[\mathrm{MHz}]$ & 36.51 & 36.32 & 36.25 \\
$A_{1}\left[\mathrm{MHz} \mathrm{K}^{-1}\right]$ & $-0.3464 \times 10^{-3}$ & $-0.2179 \times 10^{-2}$ & $-0.7818 \times 10^{-3}$ \\
$A_{2}\left[\mathrm{MHz} \mathrm{K}^{-2}\right]$ & $-0.1096 \times 10^{-5}$ & $-0.8096 \times 10^{-5}$ & - \\
$A_{3}\left[\mathrm{MHz} \mathrm{K}^{-3}\right]$ & $-0.4722 \times 10^{-8}$ & - & - \\
$A_{4}\left[\mathrm{MHz} \mathrm{K}^{-4}\right]$ & $-0.2041 \times 10^{-10}$ & - & $0.412 \times 10^{-2}$ \\
$\Delta[\mathrm{MHz}]$ & $0.231 \times 10^{-3}$ & $0.371 \times 10^{-3}$ & - \\
$\langle g\rangle\left[\mathrm{MHz} \mathrm{K}{ }^{-1}\right]$ & 0.0024 & 0.0021 &
\end{tabular}

TABLE II

Least squares fit parameters and average temperature coefficient $\langle g\rangle$ for Line 2 of 3,4-dichloro nitrobenzene.

\begin{tabular}{|c|c|c|c|}
\hline Parameters & $\begin{array}{c}4 \text { th order } \\
\text { polynomial }\end{array}$ & Brown $\left(T_{0}=200 \mathrm{~K}\right)$ & Kushida \\
\hline$A_{-1}[\mathrm{MHz} \mathrm{K}]$ & - & - & $0.468 \times 10^{2}$ \\
\hline$A_{0}[\mathrm{MHz}]$ & 37.1 & 36.81 & 36.71 \\
\hline$A_{1}\left[\mathrm{MHz} \mathrm{K} \mathrm{K}^{-1}\right]$ & $-0.6576 \times 10^{-3}$ & $-0.2953 \times 10^{-2}$ & $-0.1083 \times 10^{-2}$ \\
\hline$A_{2}\left[\mathrm{MHz} \mathrm{K}^{-2}\right]$ & $-0.1888 \times 10^{-5}$ & $-0.9760 \times 10^{-5}$ & - \\
\hline$A_{3}\left[\mathrm{MHz} \mathrm{K}^{-3}\right]$ & $-0.6010 \times 10^{-8}$ & - & - \\
\hline$A_{4}\left[\mathrm{MHz} \mathrm{K}^{-4}[\right.$ & $-0.1961 \times 10^{-10}$ & - & - \\
\hline$\Delta[\mathrm{MHz}]$ & $0.214 \times 10^{-3}$ & $0.299 \times 10^{-3}$ & $0.706 \times 10^{-2}$ \\
\hline$\langle g\rangle\left[\mathrm{MHz} \mathrm{K}^{-1}\right]$ & 0.0021 & 0.0018 & - \\
\hline
\end{tabular}

In the case of both lines, the fourth order polynomial gives the best fit and fit of Brown's equation is second best. This indicates that the temperature variation of the torsional frequencies is significant and cannot be neglected in this compound.

It is necessary to calculate the moment of inertia of the molecule before calculation of the torsional frequencies. X-ray crystal structure data was not available for 3,4-dichloro nitrobenzene in the literature but was available for the related compound 3,4,5-trichloro nitrobenzene [18]. This structure data of bond lengths and angles was used, with the modification that the chlorine on carbon- 5 was removed, to calculate the moment of inertia tensor for the molecule. The contribution of H-atoms to the moment of inertia was neglected due to their small mass. Table III gives the moment of inertia, the angles $\alpha_{i}$ that their principal axes make with the $z$-axis of the EFG and the factor $\sin ^{2} \alpha_{i} / A_{i}$ in each case.

The torsional frequencies calculated under the two-mode approximation are plotted as a function of temperature in Figs. 1 and 2. The lower frequency mode $f_{2}$ in both cases is the same and corresponds to motion about an axis perpendicular to the ring and lies roughly between $20 \mathrm{~cm}^{-1}$ and $40 \mathrm{~cm}^{-1}$. In Fig. $1, f_{1}$ corresponds to motion about an axis in plane of the ring making $101.13^{\circ}$ with the $\mathrm{C}-4 \mathrm{Cl}$ bond. This mode is close to $f_{2}$, only slightly higher. In Fig. $2, f_{1}$ corresponds to motion about axis in the plane of the ring but making an angle $-50.27^{\circ}$ with the $\mathrm{C}-3 \mathrm{Cl}$ bond. This is much higher in frequency ranging from $45 \mathrm{~cm}^{-1}$ to $75 \mathrm{~cm}^{-1}$. 


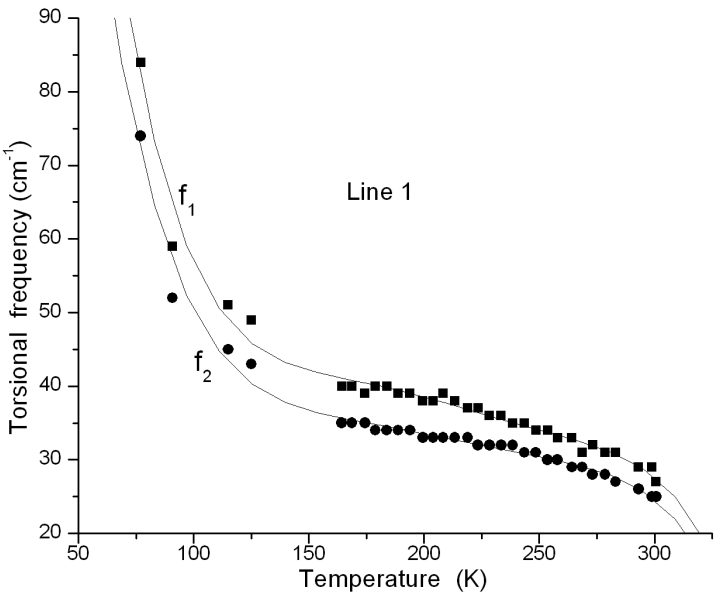

Fig. 1. Temperature dependence of torsional frequencies calculated from Line 1 (4-Cl) of 3,4-dichloro nitrobenzene. Frequency $f_{2}$ corresponds to motion about an axis perpendicular to the benzene ring while $f_{1}$ corresponds to motion about an axis making $101.13^{\circ}$ with $\mathrm{C}-4 \mathrm{Cl}$ bond in the plane of the ring. The continuous line is a guide to the eye.

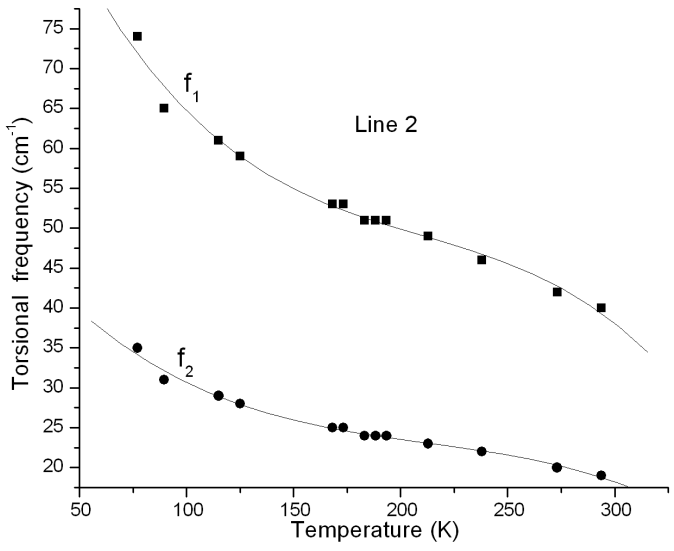

Fig. 2. Temperature dependence of torsional frequencies calculated from Line $2(3-\mathrm{Cl})$ of 3,4-dichloro nitrobenzene. Frequency $f_{2}$ corresponds to internal motion about an axis perpendicular to the benzene ring while $f_{1}$ corresponds to motion about an axis making $-50.27^{\circ}$ with $\mathrm{C}-3 \mathrm{Cl}$ bond in the plane of the ring. The continuous line is a guide to the eye.

Moments of inertia, angles $\alpha_{i}$ and weighting factors for Tatsuzaki model in 3,4-dichloro nitrobenzene.

\begin{tabular}{c|c|c|c|c|c|c}
\hline \hline$A_{i}$ & \multicolumn{2}{|c|}{$1649 \times 10^{-40} \mathrm{~g} \mathrm{~cm}^{2}$} & \multicolumn{2}{c|}{$2127 \times 10^{-40} \mathrm{~g} \mathrm{~cm}^{2}$} & \multicolumn{2}{c}{$478 \times 10^{-40} \mathrm{~g} \mathrm{~cm}^{2}$} \\
\hline & $\alpha_{1}$ & $\frac{\sin ^{2} \alpha_{i}}{A_{1}}$ & $\alpha_{2}$ & $\frac{\sin ^{2} \alpha_{2}}{A_{2}}$ & $\alpha_{3}$ & $\frac{\sin ^{2} \alpha_{3}}{A_{3}}$ \\
\hline $\begin{array}{c}\text { Line 1 } \\
\text { 4-Cl }\end{array}$ & $101.13^{\circ}$ & $5.838 \times 10^{36}$ & $90^{\circ}$ & $4.702 \times 10^{36}$ & $11.13^{\circ}$ & $7.799 \times 10^{35}$ \\
\hline $\begin{array}{c}\text { Line 2 } \\
\text { 3-Cl }\end{array}$ & $39.73^{\circ}$ & $2.477 \times 10^{36}$ & $90^{\circ}$ & $4.702 \times 10^{36}$ & $-50.27^{\circ}$ & $12.378 \times 10^{36}$
\end{tabular}

It is seen that the torsional frequencies lie in the range $25 \mathrm{~cm}^{-1}$ and $85 \mathrm{~cm}^{-1}$ in the case of Line 1 and $25 \mathrm{~cm}^{-1}$ to $75 \mathrm{~cm}^{-1}$ in the case of Line 2 . The temperature dependence is nearly linear for temperatures above $150 \mathrm{~K}$ and non-linear at temperatures below that in the case of Line 1. The torsional frequencies differ greatly and have a nearly linear temperature dependence throughout the temperature range in the case of Line 2.

\subsection{4-chloro 2 nitrophenol}

Phenols contain an electronegative $\mathrm{OH}$ group and have a tendency to form hydrogen bonds in the crystal. The effect of this is often seen in the lowering of the NQR frequency of the chlorine which is hydrogen bonded to the OH. 4-chloro 2 nitrophenol contains a nitro group along with the phenol and an attempt has been made to study the temperature dependence of the torsional frequencies in this compound.
The ${ }^{35} \mathrm{Cl}$ NQR in 4-chloro 2 nitrophenol was observed by Chakrapani et al. [19] in the temperature range 90-357 $\mathrm{K}$ and a single resonance was found throughout. The NQR frequency at room temperature was $35.125 \mathrm{MHz}$. As the molecule contains a single chlorine, the observed line can be assigned to it. Iterative least squares fit of the NQR frequencies to three Eqs. (2), (3) and (4) shows that Brown's equation gives the best fit indicating that the temperature dependence of the internal torsional frequencies is significant in this case, too. Details of this fit are recorded in Table IV with $\langle g\rangle$ and $\Delta$ being the average temperature coefficient of the torsional frequencies and root mean square deviation value, respectively, as in the previous case. Accuracy of the parameters quoted are retained to an order higher than the experiment in this case, too.

To calculate the moments of inertia to be used in Tatsuzaki equation under the two-mode approximation, 
TABLE IV Least squares fit parameters and average temperature coefficient $\langle g\rangle$ in 4-chloro 2 nitrophenol.

\begin{tabular}{l|c|c|c}
\hline \hline \multicolumn{1}{c|}{ Parameters } & $\begin{array}{c}\text { 4th order } \\
\text { polynomial }\end{array}$ & $\begin{array}{c}\text { Brown } \\
\left(T_{0}=225 \mathrm{~K}\right)\end{array}$ & Kushida \\
\hline$A_{-1}[\mathrm{MHz} \mathrm{K}]$ & - & - & $0.512 \times 10^{+2}$ \\
$A_{0}[\mathrm{MHz}]$ & 35.68 & 35.32 & 35.26 \\
$A_{1}\left[\mathrm{MHz} \mathrm{K}^{-1}\right]$ & $-0.1053 \times 10^{-2}$ & $-0.2185 \times 10^{-2}$ & $0.1013 \times 10^{-2}$ \\
$A_{2}\left[\mathrm{MHz} \mathrm{K}^{-2}\right]$ & $-0.1976 \times 10^{-5}$ & $-0.3179 \times 10^{-5}$ & - \\
$A_{3}\left[\mathrm{MHz} \mathrm{K}^{-3}\right]$ & $-0.2026 \times 10^{-8}$ & - & - \\
$A_{4}\left[\mathrm{MHz} \mathrm{K}^{-4}\right]$ & $0.1521 \times 10^{-11}$ & - & - \\
$\Delta\left[\mathrm{MHz}^{-1}\right]$ & $0.336 \times 10^{-3}$ & $0.329 \times 10^{-3}$ & $0.352 \times 10^{-2}$ \\
$\langle g\rangle\left[\mathrm{MHz} \mathrm{K}{ }^{-1}\right]$ & 0.0030 & 0.0008 & -
\end{tabular}

\section{TABLE V}

Moments of inertia, angles $\alpha_{i}$ and weighting factors for Tatsuzaki model 4-chloro 2 nitrophenol.

\begin{tabular}{c|c|c|c}
\hline \hline & $A_{i}\left[\mathrm{~g} \mathrm{~cm}^{2}\right]$ & $\alpha_{i}$ & $\frac{\sin ^{2} \alpha_{i}}{A_{i}}$ \\
\hline$i=1$ & $1286.805 \times 10^{-40}$ & $114.11^{\circ}$ & $6.474 \times 10^{36}$ \\
$i=2$ & $1722.622 \times 10^{-40}$ & $90^{\circ}$ & $5.805 \times 10^{36}$ \\
$i=3$ & $435.817 \times 10^{-40}$ & $24.11^{\circ}$ & $3.829 \times 10^{36}$
\end{tabular}

crystal structure data of the above compound is necessary. As this was not available, the crystal structure of $p$-chlorophenol [20] was used and the nitro group of standard bond lengths was added in the 2-position. The phenomenon of polymorphism has been observed in $p$-chlorophenol; i.e. it exhibits two structural forms, $\alpha$-chlorophenol and $\beta$-chlorophenol. It is seen that the

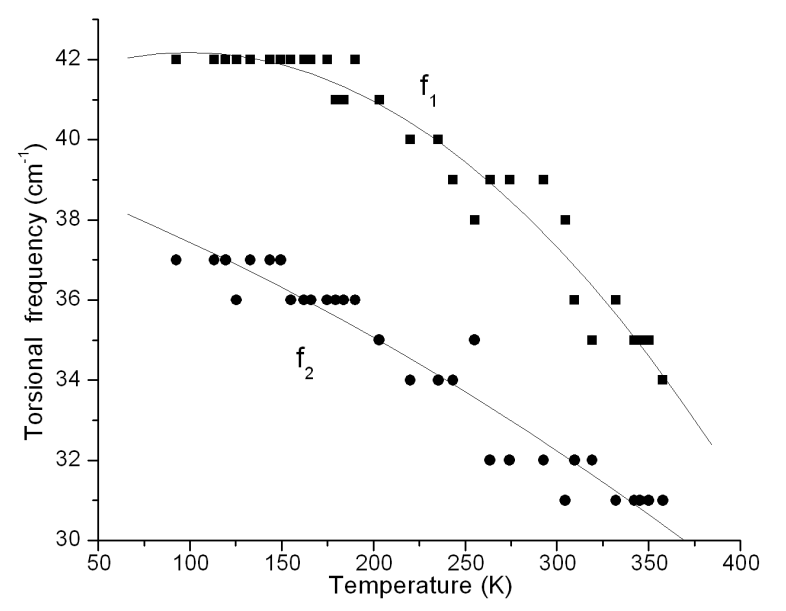

Fig. 3. Temperature dependence of torsional frequencies calculated from Line 1 (4-Cl) of 4-chloro 2 nitrophenol. 2 modes affecting the 4 -Cl frequency are $f_{2}$ corresponding to motion about an axis perpendicular to the benzene ring while $f_{1}$ corresponds to motion about an axis in the plane of the ring making an angle of $114.11^{\circ}$ with the $\mathrm{C}-\mathrm{Cl}$ bond. The continuous line is a guide to the eye. benzene ring in both forms is nearly a regular hexagon. Hence a regular hexagon was used for the benzene ring part. Table $\mathrm{V}$ shows the principal values of the moment of inertia tensor, the angles $\alpha_{i}$ that their axes make with the $z$-axis of the EFG and the weighting factor for the three terms in the Tatsuzaki equation.

The torsional frequencies were calculated under the two-mode approximation and their temperature dependence is shown in Fig. 3. The mode corresponding to motion about an axis perpendicular to the benzene ring is $f_{2}$ and has a small variation from $31 \mathrm{~cm}^{-1}$ to $37 \mathrm{~cm}^{-1}$. The mode $f_{1}$ corresponds to motion about an axis in the plane of the ring making an angle $114.11^{\circ}$ with the $\mathrm{C}-\mathrm{Cl}$ bond. The torsional frequencies are seen to lie in the small range $30 \mathrm{~cm}^{-1}$ and $42 \mathrm{~cm}^{-1}$ and the temperature variation is nearly linear in the case of both torsional frequencies. The smaller variation of the torsional frequencies with temperature may be because of hydrogen bonding. The data in Fig. 3 seems scattered as the resolution assumed in the computer for calculating the torsional frequencies is $1 \mathrm{~cm}^{-1}$. This introduces a step function like effect in the numerical data generated in this case.

\section{Conclusions}

The temperature dependence of the torsional modes affecting the NQR frequencies have been calculated, under a two-mode approximation, in the two compounds 3,4-dichloro benzene and 4-chloro 2 nitro phenol. The torsional frequencies lie between $25 \mathrm{~cm}^{-1}$ and $85 \mathrm{~cm}^{-1}$ in the case of 3,4-dichloro benzene and their temperature dependence is nearly linear above $150 \mathrm{~K}$. In the case of 4-chloro 2 nitro phenol the torsional frequencies vary much less, only between $30 \mathrm{~cm}^{-1}$ and $42 \mathrm{~cm}^{-1}$ and the variation is nearly linear in the temperature range $77 \mathrm{~K}$ to $350 \mathrm{~K}$. The reduced temperature dependence of the torsional frequencies in this case may be due to intermolecular hydrogen bonding.

\section{Acknowledgments}

K.R. acknowledges partial financial support under the COSIST program of the University Grants Commission.

\section{References}

[1] D. Biedenkapp, A. Weiss, J. Chem. Phys. 49, 3933 (1968).

[2] J.P. Bayle, J. Jullien, H. Stahl-Lariviere, L. Guibe, J. Mol. Struct. 58, 487 (1980).

[3] H. Bayer, Z. Phys. 130, 227 (1951).

[4] T. Kushida, G. Benedek, N. Bloembergen, Phys. Rev. 104, 1364 (1956).

[5] R.J.C. Brown, J. Chem. Phys. 32, 116 (1960).

[6] I. Tatsuzaki, Y. Yokozawa, J. Phys. Soc. Japan 12, 802 (1957). 
[7] K. Rukmani, J. Ramakrishna, J. Mol. Struct. 127, 149 (1985).

[8] K. Rukmani, J. Ramakrishna, J. Chem. Soc. Faraday Trans. 2 82, 291 (1986).

[9] H.D. Basavegowda, K. Rukmani, Acta Phys. Pol. A 111, 257 (2007); V S Shanthala, K Rukmani, Acta Phys. Pol. A 114, 845 (2008).

[10] S.A. Barnett, A. Johnston, A.J. Florence, A.R. Kennedy, Acta Crystallogr. E 61, 02318 (2005).

[11] S.A. Barnett, A. Johnston, A.J. Florence, A.R. Kennedy, Acta Crystallogr. E 61, 03666 (2005).

[12] T.P. Das, E.L. Hahn, NQR Spectroscopy, Solid State Physics, Supplement I, Academic Press, New York 1958.

[13] R. Clarke, D. Siapkas, J. Phys. C, Solid State Phys. 8, 377 (1975).
[14] V. Lughi, D.R. Clarke, J. Appl. Phys. 101, 053524 (2007).

[15] M.S. Vijaya, J. Ramakrishna, Mol. Phys. 19, 131 (1970).

[16] V.S.S. Sastry, J. Ramakrishna, Indian J. Pure Appl. Phys. 15, 658 (1977).

[17] J. Ramakrishna, Proc. R. Soc. Lond. 86, 595 (1965).

[18] A. Bhar, J.P. Aune, Acta Crystallogr. C 51, 256 (1995).

[19] G. Chakrapani, C.V. Rama Sastry, C.R.K. Murthy, J. Phys. C, Solid State Phys. 6, 1444 (1973).

[20] I.M. Perrin, P. Michel, Acta Crystallogr. B 29, 253 (1973). 